\title{
AUSBAU VON OPEN ACCESS AN DEN ÖSTERREICHISCHEN UNIVERSITÄTEN: BUDGETÄRER MEHRBEDARF FÜR DIE JAHRE 2019-2021. ZUSAMMENFASSUNG DES ABSCHLUSS- BERICHTS DER HRSM AT2OA-TRANSITION-STUDIE
}

\author{
von Georg Fessler
}

Zusammenfassung: Im Rahmen des vom österreichischen Bundesministerium für Bildung, Wissenschaft, Forschung geförderten HRSM-Projekts Austrian Transition to Open Access (AT2OA) wurde ein Teilprojekt etabliert, welches sich mit Budgetund Kostenfragen eines weiteren Ausbaus von Open Access an den österreichischen Universitäten beschäftigt. In einer ersten Phase des Teilprojekts wurde der Frage nachgegangen, ob ein weiterer Ausbau von Open Access in den Jahren 2019-2021 budgetäre Konsequenzen hat und - wenn ja - welche. Die Methoden und Ergebnisse dieser Transition-Studie werden im vorliegenden Artikel zusammengefasst.

Schlagwörter: Hochschulraumstrukturmittelprojekt; Austrian Transition to Open Access (AT2OA); Open Access; Transition; Transformation; Konsortien; Publikationsgebühren; AT2OA; Österreich

\section{EXPANSION OF OPEN ACCESS AT AUSTRIAN UNIVERSITIES: FINANCIAL NEEDS FOR THE YEARS 2019-2021. SUMMARY OF THE FINAL REPORT OF THE HRSM AT2OA TRANSITION-STUDY}

\begin{abstract}
Within a project commissioned by the Austrian Federal Ministry of Education, Science and Research called Austrian Transition to Open Access (AT2OA) a sub-project was established to investigate which budgetary implications, if any, a further expansion of Open Access at Austrian universities would have. In phase one of the sub-project financial needs for the years 2019-2021 were investigated. The present article summarizes the methods and findings of the final report of the AT2OA-Transition-Study.
\end{abstract}

Keywords: Higher Education Structural Funds Project; Austrian Transition to Open Access (AT2OA) Open Access; Transition; Transformation; Consortia; Article Processing Charges; AT2OA; Austria

DOI: https://doi.org/10.31263/voebm.v72i1.2275

(c) Georg Fessler

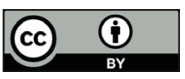

Dieses Werk ist lizenziert unter einer

Creative-Commons-Lizenz Namensnennung 4.0 International 


\section{Inhalt}

1. AT2OA-Transition-Studie

2. Unterstützende Analysen und Datensammlungen

3. Analyse der zwei wesentlichen Open Access-Kostenfaktoren

4. Resümee

\section{AT2OA-Transition-Studie}

Im Rahmen des vom österreichischen Bundesministerium für Bildung, Wissenschaft und Forschung (BMBWF) geförderten Hochschulraumstrukturmittelprojekts Austrian Transition to Open Access (AT2OA) wurde ein Teilprojekt etabliert, welches sich mit Budget- und Kostenfragen eines weiteren Ausbaus von Open Access an den österreichischen Universitäten beschäftigt. In einer ersten Phase des Teilprojekts wurde der Frage nachgegangen, ob ein weiterer Ausbau von Open Access in den Jahren 20192021 budgetäre Konsequenzen hat und - wenn ja - welche. Die Methoden und Ergebnisse dieser Transition-Studie liegen in einem Abschlussbericht vor, der hier zusammenfassend dargestellt wird ${ }^{1}$.

Die Übergangszeit, die in der „Transition-Studie“ analysiert wird, zeichnet sich dadurch aus, dass für wissenschaftliche Zeitschriften sowohl das traditionelle Subskriptionsmodell als auch neue Open Access-Finanzierungsmodelle nebeneinander bestehen. Die Bezeichnung „Übergangszeit“ geht von dem Gedanken aus, dass am Ende der Transition das gesamte wissenschaftliche Publikationswesen weltweit auf Open Access umgestellt ist und aus Budgetsicht keine Ausgaben mehr für den lesenden Zugang zu wissenschaftlicher Literatur, sondern nur mehr für das wissenschaftliche Publizieren anfallen.

\subsection{Untersuchte Themenbereiche}

Um Budgetprognosen für die Jahre 2019-2021 zu erstellen, wurden zwei wesentliche Kostenfaktoren analysiert:

1. Welche Budgets sind in den nächsten Jahren notwendig, um die bestehenden Konsortialverträge mit Open Access-Komponente weiterzuführen und/oder auszubauen?

2. Welche Mittel sind notwendig, um diejenigen Publikationen Open Access zu veröffentlichen, die bei Verlagen publiziert werden, mit denen keine Verträge mit Open Access-Komponente bestehen - ein Open Access-Publizieren also, das über die Bezahlung von Article 
Processing Charges (APCs) über einen Publikationsfonds finanziert werden muss?

Um diese Fragestellungen beantworten zu können, wurden folgende unterstützenden Analysen durchgeführt und Datensammlungen zusammengestellt:

- Analyse des Publikationsoutputs Österreichs

- Ermittlung repräsentativer Durchschnittswerte für die Höhe von APCs

- Open Access-Ausgaben von Fördergebern

- AT2OA-Förderungen

$\mathrm{Zu}$ all diesen Themen wurden Daten gesammelt und aufbereitet, Berechnungsmethoden entwickelt, Szenarien entworfen und für die 24 an AT2OA teilnehmenden Einrichtungen aufgeschlüsselt. Die Ergebnisse und Berechnungsmethoden wurden den Institutionen im Rahmen eines Workshops im April 2018 präsentiert und im Mai 2018 durch Zusendung eines einrichtungsspezifischen Datensets mit Erläuterungen sowie einer Vorlage für die Erstellung lokaler Berichte zur Verfügung gestellt.

Die AT2OA-Einrichtungen hatten dann auf lokaler Ebene zwei Optionen: Entweder die zur Verfügung gestellten Analysen als Input für ihre Budgetplanungen zu übernehmen. Diese Option wurde von den Einrichtungen gewählt, wo Open Access keinen großen budgetrelevanten Faktor darstellt oder bei Vorliegen der zentralen Daten und Analysen im Mai 2018 die Planungen für die Jahre 2019-2021 schon weitgehend abgeschlossen waren oder aus Zeit- und Kapazitätsgründen auf eine lokale Studie verzichtet werden musste. Acht Universitäten haben sich dafür entschieden, einen detaillierten lokalen Bericht auszuarbeiten. In diesen lokalen Berichten wird der Stand der Open Access-Bemühungen der Einrichtung im Detail beschrieben, zentrale Analysen wie z. B. für die Szenarien für Konsortien mit Open Access-Komponente auf die jeweilige Institution umgelegt, lokale Berechnungen vorgenommen und die daraus folgenden budgetären Konsequenzen für die jeweilige Einrichtung für die Jahre 2019-2021 erläutert.

Die Transition-Studie konzentriert sich auf die Analyse der oben beschriebenen Kostenfaktoren. Der Schwerpunkt liegt auf wissenschaftlichen Zeitschriftenartikeln, andere Publikationstypen wie z. B. Monographien wurden nicht untersucht. Durch die Benützung der Datenquellen Web of Science und Scopus ergibt sich eine Konzentration auf englischsprachige Publikationen. Wissenschaftliche Zeitschriften, in denen nicht in englischer Sprache publiziert wird, sind somit unterrepräsentiert. Andere budgetre- 
levante Aspekte von Open Access wie z. B. Infrastrukturkosten für institutionelle Repositorien oder österreichische Open Access-Journals und Förderungen alternativer, nicht-kommerzieller Open Science-Infrastrukturen und Services werden in der Transition-Studie nicht behandelt.

\section{Unterstützende Analysen und Datensammlungen}

\subsection{Publikationsoutput Österreich}

Grundlage für die Datenerhebung zum österreichischen Publikationsoutput bilden die Datenbanken Web of Science (WoS, von Clarivate Analytics) und Scopus (von Elsevier) ${ }^{2}$. Es wurden sämtliche österreichischen Publikationen in WoS und Scopus abgefragt, heruntergeladen und Rohdatenpakete im Excel-Format erstellt, die für 2016 insgesamt 50.480 Einträge (26.090 WoS, 24.390 Scopus) enthalten. Diese Rohdatenpakete wurden zunächst einer Datenbereinigung unterzogen, die 2,62\% der Daten betraf. Für die Erstellung des AT2OA-Datasets wurden die beiden Rohdatenpakete zusammengefügt. Im Ergebnis sind zu jedem Publikationseintrag sämtliche Angaben aus WoS und Scopus (soweit eine Publikation in beiden Datenbanken erfasst ist) in einer Zeile zusammengefasst. Über einen Abgleich der DOls sowie der PubMed IDs konnten rund $65 \%$ der Einträge zusammengeführt werden. Die verbliebenen Einträge wurden zur Feststellung weiterer zusammengehöriger Publikationen einem mehrstufigen Abgleichverfahren unterzogen. Sämtliche Publikationen, die in WoS und Scopus eindeutig als Buchbeiträge ausgewiesen sind, wurden aus dem AT2OA-Dataset ausgeschlossen.

Nach Abschluss der Datenbereinigung, der Datenzusammenführung sowie dem Ausschluss von Buchbeiträgen umfasst das Datenset für 2016 28.214 Publikationen (davon 18.313 Publikationen in WoS und Scopus, 4.585 Publikationen nur in WoS und 5.316 Publikationen nur in Scopus).

Die zentrale Aufgabe in der Datenaufbereitung war die Zuweisung einer oder mehrerer institutioneller Affiliationen zu jeder Publikation. Unterschieden wurde, im Fall von AT2OA relevanten Publikationen, zwischen Corresponding Author-Affiliationen und Co-Author-Affliliationen. Nicht AT2OA relevante Publikationen wurden mit den beiden Sammelaffiliationen „Austria“ bzw. „Ausland“ gekennzeichnet.

Die systematische Erfassung der AT2OA relevanten Affiliationen beruht auf einer Kategorisierung in drei Affiliationsstypen:

- Typ 1 - Publikationen, bei denen mindestens ein AT2OA-Partner als Corresponding Author erscheint. 
- Typ 2 - Publikationen, bei denen kein AT2OA-Partner Corresponding Author ist, aber mindestens ein AT2OA-Partner Co-Autor ist.

- Typ 3 -Publikationen, für die kein AT2OA-Bezug festgestellt wurde.

WoS und Scopus enthalten sechs Datenfelder, die wichtige Informationen für die institutionelle Zuweisung enthalten. In Ermangelung eines formalisierten Vokabulars für die Benennung von Universitäten und Forschungseinrichtungen stellen sich diese Felder als problematisch in der praktischen Anwendung auf Massendaten dar. Abhilfe bot hier nur die systematische Zusammenstellung einer Sammlung aus rund 600 Begriffen, die anschließend zur Feststellung der institutionellen Zugehörigkeit einer Publikation zu einer der 24 AT2OA-Partnereinrichtungen verwendet werden konnte. Dieser Glossar wurde mit den WoS- und Scopus-Angaben abgeglichen und die ermittelten Zuweisungen im Dataset erfasst.

Bezugnehmend auf die drei Typengruppen wurden im Rahmen der Institutionenzuweisung folgende Ergebnisse für 2016 ermittelt (Dataset gesamt 28.214 Publikationen):

- Typ 1: 13.792 Publikationen mit mindestens einem AT2OA-Partner als Corresponding Author

- Typ 2: 7.926 Publikationen bei denen kein AT2OA-Partner Corresponding Author ist, aber mindestens ein AT2OA Partner Co-Autor ist

- Typ 3: 6.496 Publikationen für die kein AT2OA-Bezug festgestellt wurde

Für die weiteren Berechnungen von Open Access-Kosten im Rahmen der Transition-Studie sind nur jene Publikationen relevant, bei denen mindestens ein AT2OA-Partner als Corresponding Author nachgewiesen ist (Typ 1).

WoS unterscheidet zwischen rund 40 Dokumentenarten, Scopus beschränkt deren Anzahl auf 11. Die 13.792 Publikationen des Typs 1 wurden drei Gruppen von Dokumentenarten zugeordnet: Articles \& Reviews, Conference Papers, Other Document Types, mit folgendem Ergebnis:

- Articles \& Reviews: 9.763 Publikationen

- Conference Papers: 3.231 Publikationen

- Other Document Types: 798 Publikationen

Den Abschluss der Datenaufbereitung bildete neben der Vereinheitlichung der von WoS und Scopus verwendeten Source Titles (Journal Titles) und der Vereinheitlichung der Publisher Names, die Zuweisung der Publikationen zu wissenschaftlichen Fachbereichen. Bei letzterer hat sich gezeigt, dass sich zwischen den Angaben in WoS und in Scopus, aber auch zu anderen Daten- 
beständen teils gravierende Diskrepanzen auftun, die im Rahmen dieses Projekts nur mittels einer maximalen Simplifikation zu überwinden waren. In diesem Sinn, und natürlich auf Kosten der Differenziertheit, wurden sämtliche Publikationen einer oder mehreren der folgenden drei Fachbereiche zugewiesen: Life Sciences, Natural Sciences sowie Social Sciences \& Humanities.

Nach Abschluss der Erstellung, Bereinigung und Aufbereitung des AT2OA-Datasets wurden im Rahmen einer Datenerweiterung einige relevante Datenbestände eingespielt: Informationen aus Kostenübernahmen durch den FWF, der Open APC Initiative ${ }^{3}$, der Unpaywall Database ${ }^{4}$, des Directory of Open Access Journals ${ }^{5}$, aus Ulrichsweb ${ }^{6}$ sowie die WoS-Indices Angaben.

Die Publikationsoutputdaten 2016 wurden den Einrichtungen im Mai 2018 übermittelt. Die Daten wurden soweit aufbereitet, dass sie von den einzelnen AT2OA-Partnerinstitutionen mittels unique identifiers bzw. über die Publikationstitel mit den Daten in den lokalen Forschungsdokumentationssystemen abgeglichen werden konnten. Das Datenset für den gesamten dreijährigen Zeitraum (2015-2017) ist im Frühjahr 2019 noch in Bearbeitung und wird nach Fertigstellung den Einrichtungen bereitgestellt.

Es ist eine zusammenfassende Abschlussanalyse geplant, die auch bibliometrische Auswertungen enthalten wird. Eine erst Auswertung nach einzelnen AT2OA-Einrichtungen ist im Transition-Studie-Abschlussbericht enthalten. Die Aufschlüsselung der Publikationstätigkeit der österreichischen Universitäten nach Verlagen ergibt das bekannte Bild der Dominanz der Verlage Elsevier, SpringerNature, Wiley und Taylor \& Francis mit einem Anteil von $48 \%$ der publizierten Artikel.

Mit diesen Daten liegen erstmals Detailinformationen über den gesamten in Web of Science und Scopus erfassten Publikationsoutput der österreichischen Universitäten vor. Die Verfügbarkeit eines solchen Datensets stellt eine wichtige Grundlage im Umgang mit verschiedenen wissenschaftspolitischen und planerischen Fragestellungen dar. Es wäre wünschenswert, diese Datenaufbereitung und Analysen zu institutionalisieren und über die Laufzeit des AT2OA-Projekts hinaus regelmäßig durchzuführen.

\subsection{Open Access-Ausgaben von Fördergebern am Beispiel des FWF}

Es ist gängige Praxis, dass Fördergeber die Zuteilung von Förderungen an die Verpflichtung des Fördernehmers binden, die im Rahmen ihres Projekts entstandenen Veröffentlichungen Open Access zu publizieren. Die damit 
verbundenen Kosten werden in der Regel vom Fördergeber übernommen. Wegen der dezentralen, projektbezogenen Abwicklung solcher Kostenübernahmen ist die Datenlage zu diesen Zahlungen sehr unübersichtlich. Beispielhaft sind in der Transition-Studie die für die österreichischen Universitäten relevantesten Kostenübernahmen durch den Fonds zur Förderung der wissenschaftlichen Forschung (FWF) analysiert worden.

Der FWF hat für die Jahre 2014-2017 seine Kostenübernahmen für Open Access je Einrichtung aufbereitet ${ }^{7}$. Die vom FWF zur Verfügung gestellten Daten beinhalten sämtliche für Projekte bezahlte Open AccessAusgaben aus den Jahren 2014-2017. Im Durchschnitt hat der FWF in diesem Zeitraum rd. EUR 2,3 Mio. pro Jahr an Kosten für Gold OA- und Hybrid OA-Publikationen der AT2OA-Einrichtungen übernommen.

Neben der Übernahme der Kosten für einzelne APC-Zahlungen ist der FWF auch an zwei großen Konsortien mit Open Access-Komponente Springer Compact und Wiley - mit zentralen Zahlungen beteiligt. Des Weiteren werden bei den Konsortien Taylor \& Francis und IOP Kostenübernahmen bei APCs durch den FWF von den Verlagen direkt an die Einrichtungen refundiert. Mit den Kostenübernahmen und Beteiligungen an österreichweiten Konsortien hat der FWF den Ausbau von Open Access in Österreich vorangetrieben und diverse Universitätsbudgets entlastet.

Beim FWF gibt es Überlegungen, die derzeit administrierten Publikationskosten an die Forschungsstätten zu übertragen. Vorstellbar wäre zum Beispiel, dass für geförderte FWF-Projekte pro Einrichtung ein gewisser Prozentsatz festgelegt wird, der den Open Access-Mitteln, die den Forschungsstätten zur Verfügung gestellt werden, entspricht. Das könnte in weiterer Folge bedeuten, dass sich der FWF in Zukunft nicht mehr als eigenständige Institution zentral an Konsortien beteiligt, sondern die zur Verfügung stehenden Open Access-Fördermittel des FWFs verteilt auf die Forschungsstätten in Vertragsabkommen miteinkalkuliert werden. Voraussetzung für die Dezentralisierung der FWF-Publikationskostenabwicklung ist, dass die Umstellung von Personen- (§ 26) auf Institutionenförderung (§27) vom FWF erfolgreich implementiert wird. Ob und wann diese Umstellung der Publikationskosten umgesetzt wird, ist noch nicht bekannt. Sollte der FWF die Abwicklungsmodalitäten bei der Publikationskostenverwaltung ändern, muss an den Universitäten sichergestellt werden, dass diese Mittel, die über FWF-Projekte ausgeschüttet werden, durch universitätsinterne Prozesse in diejenigen Budgets umgeleitet werden, welche die Open Access-Ausgaben für die Universität tragen.

Aus den Daten der Open Access-Zahlungen des FWF für die Jahre 2014-2017 wurde für jede Einrichtung eine Prognose der für die Jahre 
2019-2021 zu erwartenden Kostenübernahmen zur Verfügung gestellt, welche die Einrichtungen für ihre Finanzplanungen für diese Jahre berücksichtigen konnten.

\subsection{Berechnung österreichischer APC-Durchschnittswerte}

Aus den Open Access-Zahlungen des FWF der Jahre 2014-2017 wurden für Österreich repräsentative APC-Mittelwerte errechnet. Die Datensätze beinhalten alle vom FWF für österreichische Einrichtungen bezahlten APCs. Rund 4.000 Zahlungen bilden eine solide Datengrundlage für die Kalkulation von Durchschnittswerten, aufgeschlüsselt nach den Fachbereichen Life Sciences, Natural Sciences und Social Sciences \& Humanities sowie nach den Kategorien Gold- bzw. Hybrid-Open Access. Über alle Fachbereiche hinweg ergibt sich für Gold OA-APC-Zahlungen ein Durchschnittswert von EUR 1.657,-- und für Hybrid OA-APC-Zahlungen ein Durchschnittswert von EUR 2.516,--

Die Partnereinrichtungen konnten für ihre Berechnungen verschiedener Ausbaustufen eines Open Access-Publikationsfonds die Durchschnittswerte des FWF verwenden und diejenigen Werte für weitere Berechnungen heranziehen, die der Situation an der jeweiligen Einrichtung am ehesten entsprechen.

\subsection{AT2OA-Förderungen}

Im durch das BMBWF kofinanzierte Projekt AT2OA werden den Partnereinrichtungen für die Jahre 2017 bis 2020 Budgetmittel zur Förderung des Ausbaus von Open Access an den österreichischen Universitäten zur Verfügung gestellt.

Die Einrichtungen bekommen aus den Fördermitteln Zahlungen für folgende Zwecke ausgeschüttet:

- Teilprojekt 2-Mittel für die Förderung von Konsortialverträgen mit Open Access-Komponente

- Teilprojekt 3-Mittel zur Unterstützung bei der Einrichtung bzw. des weiteren Ausbaus lokaler OA-Publikationsfonds

- Teilprojekt 4-Mittel zur Förderung eigener Open Access-Initiativen und alternativer OA-Publikationsmodelle

In Summe werden über den gesamten Zeitraum EUR 2.342.548,- an die Einrichtungen ausgeschüttet und in jährlichen Tranchen zur Verfügung gestellt. Ab dem Jahr 2021 müssen diese Fördermittel aus anderen Quellen 
finanziert werden. Im letzten Förderjahr 2020 bekommen die Einrichtungen in Summe EUR 660.584,- ausbezahlt.

\section{Analyse der zwei wesentlichen Open Access-Kostenfaktoren}

\subsection{Konsortialverträge mit Open Access-Komponente}

Die österreichischen Universitätsbibliotheken haben im Rahmen der Kooperation E-Medien Österreich (KEMÖ) früh begonnen, mit großen Wissenschaftsverlagen Konsortialverträge zu verhandeln, die sowohl den lesenden Zugriff auf die Inhalte regeln als auch Möglichkeiten schaffen, Artikel von Forscherlnnen aus am Konsortium teilnehmenden Einrichtungen Open Access zu publizieren. Dabei wurden Vereinbarungen mit unterschiedlichen Open Access-Geschäftsmodellen abgeschlossen, wie Read \& Publish-, Offsetting-Deals oder Abkommen, in denen stark rabattierte APCs für die teilnehmenden Einrichtungen vereinbart wurden. Da bei diesen Verlagen ein großer Teil der Artikel österreichischer Universitäten veröffentlicht wird, ermöglichen diese Verträge eine effektive und kostengünstige Erhöhung des Open Access-Anteils. Durch das gemeinsame, österreichweite Verhandeln der Komponenten „Lesen“ und „Publizieren“ konnte eine breite Literaturversorgung sichergestellt und die Mehrkosten für Open Access in Grenzen gehalten werden. Zusätzlich zu dieser Vorgangsweise auf nationaler Ebene konnten durch die Zentralisierung der Prozesse an den einzelnen Universitäten die Ausgaben für nicht-rabattierte APC-Zahlungen, die sich über die Jahre entwickelt haben, reduziert werden. Die Publikationskosten für Open Access-Artikel im Rahmen dieser Verträge sind deutlich niedriger als die Verlagslistenpreise für APCs.

Um die Frage zu beantworten, welche budgetären Mittel in den nächsten Jahren notwendig sein werden, um die bestehenden Konsortialverträge mit Open Access-Komponente weiterzuführen und/oder auszubauen, wurden für die österreichweiten Konsortialverträge mit Open Access-Komponente der Wissenschaftsverlage SpringerNature, Wiley, Sage, Taylor \& Francis, $I O P$ und Emerald sowie für den Elsevier-Konsortialvertrag, der derzeit keine Open Access-Komponente beinhaltet, von Expertlnnen aus Bibliotheken und der Kooperation E-Medien Österreich (KEMÖ) ${ }^{8}$ verschiedene Kostenentwicklungen für die Jahre 2019-2021 berechnet.

Dabei wurde die Methode der Szenario-Technik angewendet, bei der verschiedene Varianten von Entwicklungen entworfen und berechnet werden. Das Best-Szenario beschreibt eine kostenmäßig sehr günstige, das 
Expected-Szenario eine realistisch-optimistische, und das Worst-Szenario eine mögliche, aber pessimistische Entwicklung der Kosten.

Um dem Ziel einer Unterstützung der konkreten Budgetplanungen für die Jahre 2019-2021 nachzukommen, wurden die Berechnungen darauf ausgerichtet, allfällige Mehrkosten festzustellen. Für die Ermittlung dieses budgetären Mehrbedarfs wurde davon ausgegangen, dass jährliche Kostensteigerungen von bis zu 5\% von den Einrichtungen ohne größere Probleme finanziert werden können, als Mehrkosten wurden darüber hinaus gehende Beträge ausgewiesen.

Der FWF beteiligt sich mit Stand Sommer 2018 mit zentralen Zahlungen an zwei Konsortien mit Open Access-Komponente: Springer Compact und Wiley. Für das Springer Compact-Konsortium wird angenommen, dass der FWF sich auch an einem neuen Vertrag für die Jahre 2019-2021 mit zentralen Zahlungen in derselben Größenordnung beteiligen wird. Beim WileyKonsortium wird davon ausgegangen, dass es bei einem neuen Vertrag ab dem Jahr 2021 keine zentrale Beteiligung des FWF mehr geben wird und die Konsortialteilnehmerlnnen diese Kosten dezentral budgetieren werden müssen. Bei den Konsortien Taylor \& Francis und $I O P$ werden derzeit zentrale Ko-stenübernahmen von APCs durch den FWF von den Verlagen direkt an die Einrichtungen refundiert, was deren Budget entlastet. In allen Szenarien für Taylor \& Francis und IOP wird davon ausgegangen, dass es diese Refundierungen ab 2020 nicht mehr geben wird und diese Beträge lokal zu budgetieren sind, wobei der FWF die aus FWF-Projekten für die einzelnen Forschungsstätten anfallenden Publikationskosten kompensiert.

Da die Szenarien vertrauliche Daten der AT2OA-Einrichtungen und der Konsortialverträge enthalten, sind die Szenarien im publizierten Bericht nicht im Detail dargestellt. Es sind aber alle Variablen, Ausgangswerte, Annahmen, Wechselkurse und Berechnungsmethoden für die verschiedenen Szenarien in den Projektunterlagen ausführlich dokumentiert.

Für die dezentralen Berechnungen und Berichte wurden den AT2OA-Partnereinrichtungen die Details für diejenigen Konsortien mit Open Access-Komponente zur Verfügung gestellt, an denen die jeweilige Institution teilnimmt. Um eine österreichweite einheitliche Kalkulation sicherzustellen, wurden die Szenarien von den Einrichtungen nicht mehr weiter verändert. Ob in die jeweiligen Budgetplanungen das Best-, Expected-oder Worst-Szenario aufgenommen wurde, oblag der Entscheidung der einzelnen Einrichtungen.

Zusammenfassend kann festgehalten werden: Für die Jahre 2019 und 2020 sind auch bei den Worst-Szenarien keine dramatischen Mehrkosten zu erwarten. Es zeigt sich aber, dass ab dem Jahr 2021 - je nach Szenario 
- doch beträchtliche Mehrkosten für die Universitäten zu erwarten sind. Die größten Kostenfaktoren sind der Wegfall der AT2OA-Förderungen für Wiley und allfällige Kosten für einen Elsevier-Vertrag mit Open Access-Komponente. Zusätzlich sind Ausgaben enthalten, die möglicherweise von den Einrichtungen dezentral geplant werden müssen, falls der FWF sich aufgrund der Überlegungen zur Dezentralisierung der Administration der Open Access-Mittel nicht mehr als eigenständige Institution zentral an Konsortien beteiligt. Bei Umsetzung der Dezentralisierung hängt es von der konkreten Ausgestaltung und den Bedingungen für die Mittelverwendung $a b$, ob solch eine Systemumstellung für die Universitäten kostenneutral erfolgen wird.

\subsection{Berechnungen verschiedener Ausbaustufen eines Open Access-Publikati- onsfonds}

Die Berechnungen verschiedener Ausbaustufen eines Open Access-Publikationsfonds wurden nicht österreichweit für das Gesamtprojekt erstellt, sondern von einzelnen Einrichtungen entsprechend der Situation vor Ort vorgenommen. Dafür wurden vom Projekt Berechnungsmethoden entwickelt und von den Einrichtungen die zentral zur Verfügung gestellten Publikationsoutputdaten, Durchschnittswerte für APCs und Daten aus den Szenarioberechnungen für Konsortien mit Open Access-Komponente herangezogen.

Ziel der lokalen Berechnungen war, festzustellen, welche Open AccessQuote mit dem vorhandenen Publikationsfonds erreicht werden kann und welchen zusätzlichen Finanzbedarf es für die Ausstattung eines Publikationsfonds gibt, um unterschiedliche Open Access-Ausbaustufen - wie z. B. $50 \%, 75 \%$ oder $100 \%$ - zu erreichen.

Für die Berechnung der Publikationsfonds wurde bezüglich der Anzahl der zu berücksichtigenden Publikationen und der zu verwendenden APCWerte folgendermaßen vorgegangen:

- Es ist mittlerweile gängige Praxis, dass der Corresponding Author eines Open Access-Artikels für die Bezahlung von Article Processing Charges (APCs) verantwortlich zeichnet. Die Kosten werden in der Regel von jener Einrichtung getragen, an der die/der Wissenschaftlerln primär tätig ist. Für die Analysen wurden daher nur jene Artikel berücksichtigt, bei denen eine corresponding authorship einer/es Angehörigen einer AT2OA-Partnerinstitution nachgewiesen werden konnte.

- Bei Conference Papers ist das Thema Open Access noch nicht so etabliert wie bei wissenschaftlichen Artikeln. Daher blieben in den lo- 
kalen Analysen Conference Papers in der Berechnung des Publikationsfonds unberücksichtigt.

- Für Editorial Materials, Notes, Letters werden in der Regel keine APCs verrechnet. Diese „Anderen“ Dokumenttypen blieben daher in der Berechnung des Publikationsfonds ebenfalls unberücksichtigt.

- Als APC-Werte wurden die für Österreich ermittelten APC-Durchschnittswerte aus den FWF-Analysen verwendet. Die Einrichtungen nahmen die Gewichtung der unterschiedlichen APC-Werte der drei Fachdisziplinen und der Gold OA- oder Hybrid OA-Daten entsprechend ihrer Erfahrungen vor.

- Die Publikationen bei Verlagen mit Konsortialverträgen, bei denen ein Offsetting-oder ein rabattierter APC-Wert vereinbart ist, wurden bei den Berechnungen mit den konsortialspezifischen APC-Werten berücksichtigt.

- Für Verlage mit Read \& Publish-Konsortialverträgen müssen keine Mittel im Publikationsfonds vorgesehen werden, weil das Open Access-Publizieren bereits im Rahmen des Gesamtvertrags geregelt ist.

- Von einigen Einrichtungen wurden für die Berechnungen Wachstumsraten für den Publikationsoutput und die APC-Durchschnittswerte angenommen.

Die Ergebnisse dieser Berechnungen für einzelne Einrichtungen sind in den lokalen Transition-Studien dargestellt.

Zusammenfassend ergibt sich folgendes Bild: Die Veröffentlichung des Großteils der an österreichischen Universitäten verfassten Publikationen erfolgt in traditionellen Subskriptionszeitschriften, wobei für eine Reihe von großen Verlagen Verträge mit Open Access-Komponente vorliegen. Für die verbleibenden Publikationen in Hybrid-Zeitschriften müssen für einen weiteren Ausbau APCs bezahlt werden, wobei für diese Zeitschriften zusätzlich auch Subskriptionsausgaben anfallen. Ein massiver Ausbau von Open Access über Hybrid-Zeitschriften bei Verlagen ohne Verträge mit Open Access-Komponente führt somit zu hohen Kosten und einem sogenannten Double Dipping ${ }^{9}$ der Verlage, das tunlichst vermieden werden sollte.

Über diese Strategie eine 75\% oder sogar 100\% Open Access-Quote anzustreben, würde bei den Einrichtungen zu massiven Mehrbelastungen führen. Auch deshalb, weil im analysierten Zeitraum keine substantiellen Einsparungen für Subskriptionen zu erwarten sind, durch welche die steigenden Ausgaben des Publikationsfonds finanziert werden könnte.

Bei der Veröffentlichung von Artikeln in „reinen“ Gold Open AccessJournalen kommt es im besten Fall zu keinem Double Dipping, weil sich diese 
Zeitschriften beispielsweise über APCs oder auch andere Geschäftsmodelle finanzieren.

\section{Resümee}

Die Transition-Studie beschäftigt sich mit der Übergangszeit zu Open Access, die dadurch gekennzeichnet ist, dass das traditionelle Subskriptionsmodell für den lesenden Zugang zu wissenschaftlichen Zeitschrifteninhalten weiterbesteht und sich parallel dazu verschiedene Modelle für das Open Access-Publizieren entwickeln. Wann und ob überhaupt diese Übergangsperiode abgeschlossen werden kann, hängt von vielen Faktoren ab, u. a.: Änderung der Bewertungs-, Evaluations-, Einstellungs- und Anreizsysteme im Wissenschaftssystem; Änderung des Publikationsverhaltens von Wissenschaftlerlnnen in den verschiedenen Wissenschaftsdisziplinen; Entwicklung und Akzeptanz alternativer Open Access-Publikationsformen; weltweite Durchsetzung von Open Access - um nur einige zu nennen. Diese Faktoren können nur zu einem geringen Teil von österreichischen Akteurlnnen beeinflusst werden.

Die Studie liefert keine Hinweise darauf, dass im untersuchten Zeitraum 2019-2021 substantielle Reduktionen von Subskriptionsausgaben zu erwarten sind und schon gar nicht, dass in diesen Jahren die Übergangszeit abgeschlossen sein wird. Selbst falls es gelingen sollte, z. B. bis 2025 den gesamten österreichischen wissenschaftlichen Publikationsoutput auf Open Access umzustellen, wäre die Übergangszeit damit noch nicht abgeschlossen. Schließlich nutzen „österreichische“ Autorlnnen nicht nur „österreichische“ Publikationen. Beendet wäre die Übergangszeit letztlich erst mit einer globalen Umstellung auf Open Access und dem gleichzeitigen Ende des Verlagsfinanzierungsmodells auf Basis von Subskriptionszahlungen.

Es ist vielmehr davon auszugehen, dass das Nebeneinander der beiden Modelle über einen längeren Zeitraum hinaus besteht oder sich überhaupt eine dauerhaft heterogene Landschaft von Subskriptions- und Open Access-Modellen entwickelt. Aus budgetärer Sicht würde das bedeuten, dass nicht im hinreichenden Ausmaß Subskriptionsausgaben reduziert werden können, um damit Open Access-Modelle für eine Umstellung und Förderung von Open Access zu finanzieren.

Die gleichzeitige Verfolgung der Ziele,

- die Angehörigen der Universitäten optimal mit wissenschaftlicher Literatur zu versorgen,

- den Open Access-Anteil der Publikationen der österreichischen Universtäten sukzessive zu erhöhen, 
- Strategien zu verfolgen, welche die Marktkonzentration auf dem Markt für wissenschaftliche Zeitschriften reduziert und eine vollständige Umstellung vom Subskriptions- auf ein Open Access-Modell sicherstellen und

- die Gesamtausgaben für das Lesen und Publizieren wissenschaftlicher Literatur in Summe stabil zu halten,

stellt für die österreichischen Universitäten eine budgetäre und strategische Herausforderung dar.

Unter der Prämisse, die Gesamtausgaben für Literaturversorgung und Open Access in Summe stabil zu halten, werden zum Abschluss zwei alternative, strategische Optionen für das weitere Vorgehen der österreichischen Universitäten skizziert ${ }^{10}$ :

Es wird der Weg fortgesetzt, prioritär mit jenen Verlagen Read \& Publish-(oder ähnliche) Vereinbarungen abzuschließen und zu verlängern, bei denen die meisten österreichischen Artikel publiziert werden. Mit Wiley, SpringerNature, Taylor \& Francis, Sage, IOP und Emerald liegen solche Verträge schon vor. Eine ähnliche Vereinbarung mit Elsevier würde die Open Access-Quote der AT2OA-Einrichtungen um rund $20 \%$ erhöhen. In weiterer Folge könnten mit jenen Verlagen Verträge mit Open Access-Komponente abgeschlossen werden, deren Journals bevorzugt von österreichischen Forscherlnnen für Publikationen genutzt werden. Der „Freikauf“ einzelner Artikel über Publikationsfonds in Gold- oder Hybrid-Zeitschriften findet ergänzend statt, es wird aber über diesen Weg nicht versucht, die Open Access-Quote auf $100 \%$ zu erhöhen. Dieser Weg erscheint als effizient in der Administration und gleichzeitig als kostensparendste Methode zur Erhöhung der Open Access-Quote österreichischer Universitäten - dies bei gleichzeitiger Beibehaltung der Literaturversorgung auf hohem Niveau. Er ist jedoch umstritten, weil durch solche Big-Deals die Vormachtstellung der großen Wissenschaftsverlage weiter ausgebaut und die Marktkonzentration gefördert wird. Es wird dadurch das Hybridmodell gefestigt, ein „Flippen“ des Gesamtsystems in Richtung Open Access wird unwahrscheinlicher und die „Übergangszeit" prolongiert.

Eine alternative Strategie wäre es, bei Hybridzeitschriften auf Konsortialverträge mit Open Access-Komponente zu verzichten. Die Verträge mit Wiley, SpringerNature, Taylor \& Francis, Sage, IOP und Emerald werden in Subskriptionsverträge für Zeitschriften(teil)pakete zurückgeführt bzw. es werden überhaupt keine österreichweiten Konsortien (Big-Deals) mit Verlagen mehr abgeschlossen. Die Literaturversorgung erfolgt über lokale Einzelabonnements. Dadurch würde es zu einem Einbruch bei den Open 
Access-Publikationen in Hybrid-Zeitschriften kommen, die Open AccessQuote der österreichischen Universitäten würde sinken. Der Verzicht auf Big-Deals für Zeitschriftenpakete verschlechtert die Literaturversorgung der österreichischen Universitäten, ermöglicht aber das Umlenken von Geldern in „reine“ Open Access-Initiativen. Die österreichischen Universitäten könnten sich auf die Förderung und Finanzierung von „reinen“ Gold Open Access-Journals konzentrieren und ausschließlich Gold-APC-Zahlungen übernehmen, die Gründung neuer Open Access-Journals bzw. das Flipping von Subskriptionszeitschriften unterstützen sowie institutionelle Repositorien und alternative, nicht-kommerzielle Open Access-Infrastrukturen und Services fördern. Ob, wann und zu welchen Kosten diese Strategie die Marktkonzentration der Wissenschaftsverlage schwächt, es zu einem Anstieg der österreichischen Open Access-Quote kommt bzw. eine Gesamtumstellung auf Open Access herbeigeführt wird, ist umstritten.

Mag. Georg Fessler Wirtschaftsuniversität Wien, Universitätsbibliothek E-Mail: georg.fessler@wu.ac.at

1 Georg Fessler \& Lothar Hölbling (2019). Ausbau von Open Access an den österreichischen Universitäten: Budgetärer Mehrbedarf für die Jahre 2019-2021. Zenodo. http://doi.org/10.5281/zenodo.2621015

2 Die Publikationsoutput-Analyse inkl. Erstellung, Bearbeitung, Aufbereitung und Ergänzung der Daten wurde von Lothar Hölbling durchgeführt.

3 Open APC Initiative: https://www.intact-project.org/openapc/

4 Unpaywall: https://unpaywall.org/

5 Directory of Open Access Journals: https://doaj.org/

6 Ulrichsweb: http://www.ulrichsweb.com/

7 Die Aufbereitung der Daten erfolgte dankenswerter Weise durch Katharina Rieck vom FWF.

8 Die Szenarien wurden von Georg Fessler, Ingrid Haas, Brigitte Kromp, Karlo Pavlovic, Erika Pörnbacher und Ute Sondergeld erarbeitet.

9 Unter Double Dipping wird bei Hybrid-Zeitschriften die gleichzeitige Finanzierung von Zeitschriften sowohl über Subskriptionszahlungen als auch über APC-Zahlungen für selektive Artikel bezeichnet.

10 Der vieldiskutierte Plan S der Coalition S (https://www.coalition-s.org) wurde nach Abschluss der Arbeiten an der Transition-Studie publiziert und wird daher in diesem Bericht nicht behandelt. 